\title{
Didaktisch orientierte Rekonstruktion von Mathematik als Basis von Schulmathematik und Lehrerbildung - Editorial
}

\author{
Rolf Biehler • Werner Blum
}

Eingegangen: 21. Mai 2016 / Angenommen: 25. Mai 2016 / Online publiziert: 20. Juni 2016 (C) GDM 2016

Das vorliegende JMD-Heft verdankt sein Entstehen verschiedenen Faktoren. Es wurde in den vergangenen Jahren gelegentlich beklagt, dass im JMD empirisch orientierte Arbeiten dominieren, hingegen die Tradition fachbezogener didaktischer Analysen und Konzepte (die „Stoffdidaktik“) nicht adäquat repräsentiert ist. Dies entsprach aber keiner „Politik“ des JMD, sondern spiegelte schlicht die Orientierung der eingereichten Arbeiten wieder. Im Herausgeberteam wurde deshalb schon länger über Maßnahmen diskutiert, um potentielle Autorinnen und Autoren stärker dazu zu ermuntern, auch solche fachbezogenen didaktischen Analysen einzureichen. Die Tatsache, dass der ICME-Kongress 2016 nach 40 Jahren erneut in Deutschland stattfindet, bot einen weiteren Anlass, darüber nachzudenken, wie man entsprechende Forschungen, die ja eine lange Tradition im deutschsprachigen Raum haben und zweifellos einen Schwerpunkt der mathematikdidaktischen Arbeit bei uns darstellen, stärker im JMD repräsentieren könnte.

In diese Überlegungen fiel, dass im Oktober 2013 Arnold Kirsch im Alter von 91 Jahren verstarb. Beide Heftherausgeber waren über viele Jahre seine Kollegen in Kassel, der zweitgenannte hat schon seit den 70er Jahren eng mit ihm zusammengearbeitet. Kirschs wichtigstes Anliegen war es, Lernenden mathematische Gegenstände (Begriffe, Verfahren, Methoden, Resultate) einzeln und in größeren Einheiten so zugänglich zu machen, dass sie „Mathematik wirklich verstehen“ können (so der programmatische Titel von Kirschs wichtigstem Buch, erschienen bei Aulis 1987, zweite Auflage 1994). Eine wesentliche Methode ist dabei die fachdidaktische Analyse dieser Gegenstände.

\footnotetext{
R. Biehler $(\bowtie)$

Universität Paderborn, Warburger Str. 100, 33098 Paderborn, Deutschland

E-Mail: biehler@math.upb.de

W. Blum

Universität Kassel, Heinrich-Plett-Str. 40, 34132 Kassel, Deutschland

E-Mail: blum@mathematik.uni-kassel.de
} 
So entstand die Idee, ein Heft des JMD mit dem Themenschwerpunkt „Didaktisch orientierte Rekonstruktion von Mathematik als Basis von Schulmathematik und Lehrerbildung“ herauszugeben, gezielt dafür Beiträge einzuwerben und es Arnold Kirsch zu widmen. Die Formulierung des Themenschwerpunktes wurde gewählt, um einen möglichst allgemeinen Rahmen für die Beiträge abzustecken.

Arnold Kirschs Arbeiten zielten darauf ab, mathematische Gegenstände so aufzubereiten, dass natürliche Zugänge, wesentliche Grundvorstellungen und typische Arbeitsweisen sichtbar werden und sich idealtypische Lernsequenzen herauskristallisieren, sowohl zu einzelnen Themen (z. B. zu ganzen Zahlen, proportionalen Funktionen oder dem Integralbegriff, um nur drei von Kirschs Arbeitsgebieten zu nennen) als auch zu ganzen Stoffgebieten (z. B. zu den Funktionen, der Geometrie oder der Analysis) oder zu Arbeitsmethoden (z. B. zum Beweisen oder Modellieren). Dies war Kirsch ebenso wichtig wie die Inhalte selbst. Diese Lernsequenzen müssen nicht der tatsächlichen Entstehungsgeschichte der Gegenstände entsprechen, aber sie sollen mit didaktischer Intention so rekonstruiert werden, dass die Lernenden einen tiefen Einblick in die jeweiligen mathematischen Themen, Stoffgebiete oder Arbeitsmethoden bekommen. Solche didaktischen Rekonstruktionen bilden den Kern dessen, was man „Stoffdidaktik“ nennt. Arnold Kirsch hat dazu selber zahlreiche fundamentale Beiträge geleistet, sowohl durch seine stoffdidaktischen Analysen als auch durch allgemeine Überlegungen zum Zugänglichmachen von Inhalten, gipfelnd in seinem Plenarvortrag zum Thema „Vereinfachen im Mathematikunterricht“ (im Sinne des Zugänglichmachens für Lernende) 1976 bei der ICME-3 in Karlsruhe. Insofern dieses JMD-Heft in dem Jahr erscheint, in dem zum zweiten Mal nach 1976 ein ICME-Kongress in Deutschland stattfindet, erinnert es auch an den Mathematikdidaktiker, der als erster Deutscher zu einem solchen Plenarvortrag eingeladen war.

Das vorliegende Heft enthält neun Beiträge, die sich alle mit der didaktischen Rekonstruktion von mathematischen Gegenständen beschäftigen. Die Arbeiten stehen, ganz gemäß der Intention des Heftes, alle in der Tradition von Arnold Kirsch oder anderen „Stoffdidaktikern“. Die Beiträge stammen von verschiedenen Generationen von Mathematikdidaktikerinnen und Mathematikdidaktikern mit einer Altersspannweite von über 50 Jahren. In fünf der Beiträge geht es primär um die Rekonstruktion mathematischer Stoffinhalte: Bei Griesel um die Theorie des Messens und den GröBenbegriff, bei Buchholtz, Schwarz und Kaiser um die „Schlussrechnung“, also die proportionalen Zuordnungen, bei Oldenburg um Differentiale, bei Th. Jahnke um den Satz von Arrow und bei Engel um Datenanalyse und Funktionen. Im letztgenannten Beitrag spielt auch eine mathematische Arbeitsmethode eine zentrale Rolle, oder wie man heute auch sagen würde, eine allgemeine mathematische Kompetenz, nämlich das datengestützte funktionale Modellieren. Solche Kompetenzen stehen bei zwei weiteren der Beiträge im Mittelpunkt: Bei Biehler und Kempen das Beweisen und bei Hock, Heitzer und Schwank das axiomatische Arbeiten. Allmendinger analysiert in ihrem historisch orientierten Beitrag Kleins „Elementarmathematik vom höheren Standpunkt“ als frühes Beispiel einer Rekonstruktion von Mathematik. Schließlich wird im Beitrag von Prediger und Hefendehl-Hebeker die didaktische Rekonstruktion in das Konstrukt der epistemologischen Bewusstheit eingebettet, das eine zentrale Facette des nötigen Professionswissens von Lehrkräften beschreibt, wenn diese ihren 
Lernenden wirkliches Verstehen ermöglichen möchten. Auch diese Arbeit bezieht sich ebenso wie alle anderen explizit auf das Werk von Arnold Kirsch, dem auch die Lehrerbildung sehr am Herzen lag, denn wirkliches Verstehen kann nur eine Lehrkraft bewirken, die - so schreibt Kirsch - fachlichen Durchblick und stofflichen Überblick, die Fähigkeit zur Analyse und Reflexion der Inhalte (also genuin fachdidaktische Qualifikationen) sowie ein aktives und positives Verhältnis zum Fach besitzt.

Im Folgenden beschreiben wir kurz den Inhalt der neun Beiträge. Im Beitrag von Heinz Griesel „Die Vergleichstheorie des Messens und ihre Anwendung in der mathematikdidaktischen Grundlagenforschung" wird die Vergleichstheorie als Grundlage für die Idee des Messens dargestellt, auch in Abgrenzung von der Repräsentationstheorie des Messens. Auf Basis eines präzisierten Größenbegriffs wird Messen als multiplikatives Vergleichen von Größenwerten definiert. U. a. wird dann auch der Begriff der Proportionalität von Größen geklärt, ebenso wie der Modellbegriff. Der Autor zielt mit diesen Analysen auch bewusst darauf ab, einen Beitrag zur didaktischen Grundlagenforschung zum anwendungsorientierten Mathematikunterricht zu leisten.

Der Beitrag „Eine Analyse der sogenannten Schlussrechnung - Die Relevanz der Ansätze von Arnold Kirsch für aktuelle Lernprozesse in der Lehrerausbildung" von Nils Buchholtz, Björn Schwarz und Gabriele Kaiser knüpft an Kirschs Analysen zu proportionalen Zuordnungen an, die zweifellos zu seinen bekanntesten und einflussreichsten Arbeiten gehören. Die Autorin und die Autoren berichten über eine Untersuchung mit Lehramtsstudierenden, deren Aufgabe es war, Schülerlösungen zu Aufgaben aus der Prozentrechnung mit den Kirsch'schen Begrifflichkeiten zu analysieren, insbesondere im Hinblick auf die erkennbaren Grundvorstellungen.

Reinhard Oldenburg stellt mit seiner Arbeit „Differentiale als Prognosen - Eine Grundvorstellung als Ausgangspunkt analytischer Begriffsbildung" einen neuen stoffdidaktischen Vorschlag für den Analysisunterricht vor, der auf einem intuitiven Verständnis von Differentialen als Prognosen - und nicht als ,unendlich kleinen Größen“ - beruht. Der Vorschlag soll neue Zugänge zu Begriffsbildungen und Anwendungen der Analysis im Unterricht ermöglichen und bekannte Schwierigkeiten vermeiden.

Thomas Jahnkes Arbeit „Mathematik der Partizipation: Der Satz von Arrow Ein stoffdidaktischer Vorschlag" bereitet das berühmte, mit einem Nobelpreis gewürdigte Unmöglichkeitstheorem so auf, dass es einschließlich seines Beweises auf Schulniveau zugänglich wird. Der Satz sagt bekanntlich aus, dass gewisse vernünftig erscheinende Bedingungen an Präferenzwahlen miteinander unverträglich sind. Der Autor zeigt auf, inwiefern dieses Beispiel dem Allgemeinbildungsanspruch des Mathematikunterrichts genügen kann.

Joachim Engel beschäftigt sich mit dem Thema „Funktionen, Daten und Modelle: Vernetzende Zugänge zu zentralen Themen der (Schul-)Mathematik“. Ein wichtiges Anliegen des Beitrags ist es, die Didaktik des Modellierens und des Funktionsbegriffs stärker mit der Didaktik der Stochastik, insbesondere ihren datenorientierten Zugängen, zu vernetzen. Dazu werden beim Modellieren mit Funktionen daten- und strukturorientierte Zugänge unterschieden und zentrale Grundideen anhand tragfähiger Beispiele herausgearbeitet. 
Im Beitrag „Didaktisch orientierte Beweiskonzepte - Eine Analyse zur mathematikdidaktischen Ideenentwicklung" von Rolf Biehler und Leander Kempen werden zentrale Beweiskonzepte aus der Mathematikdidaktik, darunter ,,inhaltlich-anschauliches“, „operatives“, „,präformales“ oder ,generisches“ Beweisen, unter einer ideengeschichtlichen Perspektive analysiert und rekonstruiert. Zu den Analysekriterien gehören die jeweils verwendeten Darstellungssysteme, die Intentionen der jeweiligen Konzepte und vor allem auch, inwiefern diese Beweise Allgemeingültigkeit beanspruchen und auch im üblichen mathematischen Sinne „Beweise“ sind.

Tobias Hock, Johanna Heitzer und Inge Schwank behandeln „Axiomatisches Denken und Arbeiten im Mathematikunterricht" und plädieren für eine exemplarische unterrichtliche Berücksichtigung als wesentliches Element eines adäquaten Bildes von Mathematik. Der Artikel stellt die theoretischen Grundlagen für ein Designexperiment in der Sekundarstufe II dar. Unter Aufarbeitung wissenschaftstheoretischer Positionen zur Axiomatik in der Mathematik und der Diskussionen in der Mathematikdidaktik zur Rolle der Axiomatik (im Rahmen der so genannten „Neuen Mathematik“) wird ein neuer Vorschlag entwickelt und am Beispiel der Stochastik konkretisiert.

Der Beitrag von Henrike Allmendinger hat „Die Didaktik in Felix Kleins, Elementarmathematik vom höheren Standpunkte aus " zum Thema. Mit den Mitteln heutiger didaktischer Kategorien werden Kleins Positionen für eine „lehramtsgerechte“ Mathematik rekonstruiert und im Kontext der damaligen Situation interpretiert. Der Schwerpunkt des Artikels wird dabei auf die (implizite) Didaktik gelegt, die in Kleins Vorlesung in besonderem Maße zur Geltung kommt. Der Vergleich mit aktuellen mathematikdidaktischen Konzepten liefert Anhaltspunkte für eine fundierte Bewertung und mögliche Adaption des Klein'schen Konzepts.

Susanne Prediger und Lisa Hefendehl-Hebeker adressieren in ihrem Beitrag ,Zur Bedeutung epistemologischer Bewusstheit für didaktisches Handeln von Lehrkräften" eine wichtige Komponente des Professionswissens von Lehrkräften. Dieses Konstrukt soll alle für Lehrkräfte in didaktischen Situationen relevanten epistemologischen Aspekte erfassen. Mit der Methode der Anforderungsanalyse werden didaktische Kernaufgaben bzgl. ihrer epistemologischen Anforderungen untersucht und so wichtige Facetten dieses Konstrukts herausgearbeitet.

Alle Autorinnen und Autoren widmen ihre Beiträge Arnold Kirsch. Die von ihm wesentlich mitgeprägte „Stoffdidaktik“ zeigt sich in diesem Heft weiterhin lebendig, aber durchaus heterogen, einerseits traditionsverbunden, aber andererseits auch mit neuen Ansätzen, nicht isoliert, sondern oft verknüpft mit zugehörigen empirischen Studien. Die Frage ,Was ist Stoffdidaktik und welche Bedeutung hat sie?“ zu klären, bedarf allerdings weiterer theoretischer Anstrengungen. Hierzu sei auch auf das ebenfalls 2016 erscheinende englischsprachige JMD-Themenheft zur „Subject matter didactics“" verwiesen. Die hier vorliegende Sammlung von deutschsprachigen Aufsätzen liefert neue Beispiele, die bei der Klärung dieser Frage, so hoffen wir, eine angemessene Berücksichtigung finden werden.

Paderborn/Kassel, im Mai 2016 Rolf Biehler \& Werner Blum Gastherausgeber 